\title{
QCD motivated subtractions in hard photonic and mesonic reactions
}

\section{IInur Gabdrakhmanov*}

E-mail: ilnur@theor.jinr.ru

Bogoliubov Laboratory of Theoretical Physics, JINR, Dubna 141980, Russia

\section{Oleg Teryaev}

E-mail: teryaevetheor.jinr.ru

Bogoliubov Laboratory of Theoretical Physics, JINR, Dubna 141980, Russia

We investigate the qualitative influence of subtraction constants vector mesons electroproduction at moderate c.m.s. energies $W$ (CLAS [1], CORNELL, HERA [2, 3], E-665 [4] and HERMES [5] data). The considered subprocess is $\gamma^{*}+p \rightarrow \rho^{0}+p$ at large photon virtuality $Q^{2}$ when photon and $\rho^{0}$ meson are polarized longitudinally.

XXI International Baldin Seminar on High Energy Physics Problems September 10-15, 2012

JINR, Dubna, Russia

\footnotetext{
* Speaker.
} 


\section{Introduction}

Handbag approximation allow one to calculate meson electroproduction by convolution of the hard scattering perturbative amplitude with phenomenological functions: GPD (generalized parton distribution) and the meson DA (distribution amplitude) characterizing nonperturbative partonic content of the target nucleon and the meson respectively (for detailed reviews see [6, 7, 8]). It was shown [9] that in the handbag approximation the dominant contribution to the process is the case when both virtual photon and produced vector meson have longitudinal polarization. Other terms are suppressed by inverse powers of $Q^{2}$.

In the high $W$ (low $x_{B}$ ) region the gluon and sea quarks give the dominant contribution, while at low $W$ (high $x_{B}$ ) valence quarks give significant contribution.

A typical quark contribution handbag diagram is presented at the Fig. (1).

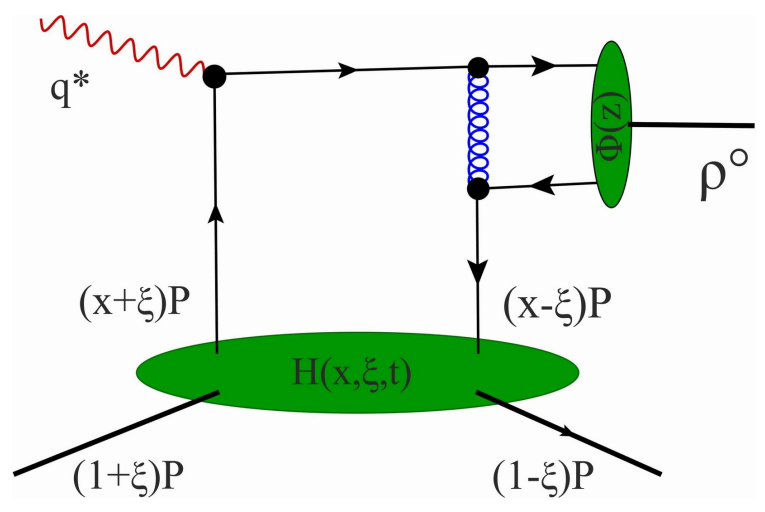

Figure 1: Leading order $\rho^{0}$ handbag quark contribution

The valence quark contribution of the collinear amplitude of the longitudinal $\rho^{0}$ electroproduction with retained proton helicity is given by [10]:

$$
A_{\text {collinear }}^{\text {val }}=\int_{-1}^{1} d x \frac{1}{\sqrt{2}}\left(e_{u} H^{u}(x, \xi)-e_{d} H^{d}(x, \xi)\right)\left\{\frac{1}{x+\xi-i \varepsilon}+\frac{1}{x-\xi+i \varepsilon}\right\}
$$

\section{Effect of the D-term}

In the paper [11] dispersion relation - holographic sum rule, connecting real and imaginary parts of the exclusive DVCS amplitudes was derived, that allows one to restore the real part from imaginary just with the use of subtraction constant. This subtraction arises from the so-called D-term (Polyakov-Weiss term [12]), containing information about t-channel zero-spin exchanges. The relation was checked analytically for the case of known in the leading order electromagnetic GPDs of the photon [13].

Goloskokov and Kroll constructed parametrization for GPDs and calculated [14, 15] cross section for longitudinal vector mesons electroproduction in the handbag approximation at the leading order. The so-called modified perturbative approach was used which takes into account transverse momenta of the partons. 
Usual way to construct a GPD parametrization (which in particular was used in mentioned work) is to obtain them from double distributions (DD) parametrization.

$$
H(z, \xi)=\int_{-1}^{1} d \alpha \int_{-1+|\alpha|}^{1-|\alpha|} d \beta f(\beta, \alpha) \delta(z-\beta-\alpha \xi)
$$

It has an important advantage of automatic abidance of the polynomiality condition $[6,7,8]$. The standard DD ansatz is:

$$
f_{i}(\beta, \alpha)=h_{i}(\beta) \frac{\Gamma\left(2 n_{i}+2\right)}{2^{2 n_{i}+1} \Gamma^{2}\left(n_{i}+1\right)} \frac{\left[(1-|\beta|)^{2}-\alpha^{2}\right]}{(1-|\beta|)^{2 n_{i}+1}}
$$

Where for $h_{i}(\beta)$ particularly in [14] the expansion was employed:

$$
h_{i}(\beta)=\beta^{-\delta_{i}}(1-\beta)^{2 n_{i}+1} \sum_{j=0}^{3} c_{i j} \beta^{j / 2}
$$

Both GK and VGG (M. Vanderhaeghen, P. A. Guichon, and M. Guidal [16]) parameterizations applied to $\rho^{0}, \rho^{+}$and $\omega$ (the ones that stand out by the presence of the contribution of valence quarks $u$ and $d$ ) mesons provide good agreement with experimental data at high energies, but lead to decreasing of the cross section with decreasing of $W \lesssim 10 \mathrm{GeV}$ while experimental data demonstrate significant growth. It was proposed in [17] to add "generalized" D-term like contribution to GPD in the form of VGG parametrization in order to cure such a behavior.

In current work we add the conventional D-term to the GK parametrization. Namely we hold first term in the Gegenbauer decomposition of the D-term:

$$
D^{q}(\alpha)=\left(1-z^{2}\right) \sum_{i=0}^{\infty} d_{i} C_{2 i+1}^{3 / 2}
$$

with coefficient obtained from chiral quark soliton model [18]:

$$
d_{0}^{C Q M}=d_{0}^{u}=d_{0}^{d}=-\frac{4.0}{N_{f}}
$$

For simplicity we use an assumption that taking into account transverse momenta of the proton and the produced meson doesn't have crucial influence on the ratio of cross sections with subtraction and without. Hence we made calculations in the collinear approximation:

$$
A_{\text {collinear }}(W)=A_{\text {collinear }}^{\text {val }}(W)+A_{\text {collinear }}^{\text {sea }}(W)+A_{\text {collinear }}^{g}(W),
$$

where valence contribution gets addition due to D-term:

$$
A_{\text {collinear }}^{\text {val }}(W) \rightarrow A_{\text {collinear }}^{\text {val }}(W)+\Delta(W),
$$

where

$$
\Delta(W)=\int_{-\xi}^{\xi} d x \frac{1}{\sqrt{2}}\left(e_{u}-e_{d}\right) D\left(\frac{x}{\xi}\right)\left\{\frac{1}{x+\xi}+\frac{1}{x-\xi}\right\}
$$


Mentioned holographic sum rule states that subtraction does not depend on $W$. At the same time as it was shown [19] the subtraction is also weakly dependent on photon virtuality $Q^{2}$.

$$
\sigma(W) \approx \sigma_{0}(W)\left|\frac{A_{\text {Collinear }}(W)+a \cdot \Delta}{A_{\text {Collinear }}(W)}\right|^{2}
$$

As we don't know how does subtraction depend on transverse degrees of freedom we added factor $a$ in the subtraction characterizing its possible dependence on transverse motion and squared transferred momentum $t$. It is known that taking into account the transverse degrees of freedom gives cross section rise for an order of magnitude. So it is reasonable to expect coefficient about $\sqrt{10} \approx 3$.

Numerical input for calculations is following: $\left\langle Q^{2}\right\rangle=4 \mathrm{GeV}, N_{f}=5$ and $t=0$. Data points and original $\sigma_{0}(W)$ dependence were taken from [15].



Figure 2: Longitudinal cross section of $\rho^{0}$ with subtraction multiplied by 1 - blue dashed, 3 - green dashdotted and fitted value $a \approx 4.8$ - red thin solid line, original GK model - black thick solid line;

Data points: CLAS - blue disks, CORNELL - red squares, HERMES - black diamonds, E665 - magenta up triangles, ZEUS - green down triangles, H1 - brown circles.

The Fig. (2) shows that subtraction with factor $a=1$ (blue dashed line) is not enough to explain discrepancy between the experimental data and the parametrization. Green dashed line corresponds to the case $a=3$ (rough approximation of transverse motion influence). The best fit with coefficient $a \approx 4.8$ is illustrated by thin red solid line.

\section{Conclusion}

We made qualitative analysis of the possible influence of the D-term on vector meson electroproduction. Several simplifications were made during the calculations. Namely collinear approximation was used and transferred momentum $t$ was set to zero. In order to compensate the loss of $t$ 
and $p_{T}$ dependence we put a numerical factor to the subtraction. The fitted factor $a \approx 4.8$ gives the reasonable agreement with the experimental points. Although subtraction has huge impact on cross section it is insufficient to explain current experimental data, therefore the other modifications of GPDs at large $x_{B}$ may be important.

The possible physical interpretation may be the following. While at high c.m.s. energies $W$ valence contribution is negligible, at the energies below $10 \mathrm{GeV}$ the valence GPD and particularly D-term have huge impact on cross section.

The latest experimental data shows that the same discrepancy arises for the $\omega$ and $\rho+$ mesons. As regards the $\rho+$, it seems possible to connect discrepancy with the D-term only if we admit the different D-terms for $u$ and d quarks.

\section{Acknowledgments}

The authors would like to thank S.V. Goloskokov, P. Kroll and D. Mueller for useful comments. I.G. is indebted to E.S. Kokoulina for discussion on fitting technique. The work is supported by RFBR grants 12-02-00613-à and 11-02-01454-à. 


\section{References}

[1] J. Santoro et al., Phys.Rev. C78, 025210 (2008).

[2] J. Breitweg et al., Eur.Phys.J. C6, 603 (1999).

[3] C. Adloff et al., Eur.Phys.J. C13, 371 (2000).

[4] M. Adams et al., Z.Phys. C74, 237 (1997).

[5] A. Airapetian et al., Eur.Phys.J. C62, 659 (2009).

[6] K. Goeke, M. V. Polyakov, and M. Vanderhaeghen, Prog.Part.Nucl.Phys. 47, 401 (2001).

[7] A. Belitsky and A. Radyushkin, Phys.Rept. 418, 1 (2005), dedicated to Anatoly V. Efremov on occasion of his 70th anniversary.

[8] M. Diehl, Phys.Rept. 388, 41 (2003), habilitation thesis.

[9] J. C. Collins, L. Frankfurt, and M. Strikman, Phys.Rev. D56, 2982 (1997).

[10] M. Vanderhaeghen, P. A. Guichon, and M. Guidal, Phys.Rev.Lett. 80, 5064 (1998).

[11] O. Teryaev, arXiv:hep-ph/0510031 (2005).

[12] M. V. Polyakov and C. Weiss, Phys.Rev. D60, 114017 (1999).

[13] I. Gabdrakhmanov and O. Teryaev, Phys.Lett. B716, 417 (2012).

[14] S. Goloskokov and P. Kroll, Eur.Phys.J. C50, 829 (2007).

[15] S. Goloskokov, Nucl.Phys.Proc.Suppl. 219-220, 185 (2011).

[16] M. Vanderhaeghen, P. A. Guichon, and M. Guidal, Phys.Rev. D60, 094017 (1999).

[17] M. Guidal and S. Morrow, Eur.Phys.J. A39, 5 (2007).

[18] V. Y. Petrov et al., Phys.Rev. D57, 4325 (1998).

[19] I. Anikin and O. Teryaev, Phys.Rev. D76, 056007 (2007). 